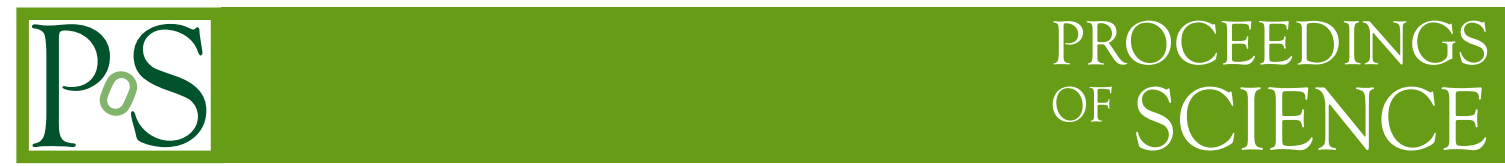

Jets as a probe of the quark-gluon plasma

\author{
Jasmine Brewer ${ }^{a, b, *}$ \\ ${ }^{a}$ Center for Theoretical Physics, Massachusetts Institute of Technology, \\ 77 Massachusetts Avenue, Cambridge, USA \\ ${ }^{b}$ Theoretical Physics Department, CERN, CH-1211 Genève 23, Switzerland \\ E-mail: jasmine.brewer@cern.ch
}

The suppression and modification of high-energy processes, like jets, in heavy-ion collisions provides an important window to access the degrees of freedom of this exotic material on different length scales. Despite increasingly precise and differential measurements of the properties of jets in heavy-ion collisions, however, it has remained challenging to use jets to make unambiguous and model-independent statements about the quark-gluon plasma. Here I will give a personal take on some origins of these challenges, including the difficulty of modelling and biases from jet selection that obfuscate the direct interpretation of jet modification measurements. I will discuss a few model studies that have helped to disentangle the source of non-intuitive effects in measurements, and finally highlight data-driven approaches as an interesting opportunity toward studying the quark-gluon plasma in a model-independent way.

HardProbes 2020

1-6 June 2020

Austin, Texas

\footnotetext{
${ }^{*}$ Speaker
} 
High-energy collisions between large nuclei provide unique experimental access to a novel hightemperature, deconfined phase of matter, the quark-gluon plasma, produced in these collisions. Though the observation of collective behavior of the quark-gluon plasma suggests that it is a strongly-coupled liquid around the scale of its temperature, at high enough momentum-transfer QCD describes quarks and gluons that are weakly-coupled. Understanding how a strongly-coupled quark-gluon plasma emerges from QCD, and how its interactions and degrees of freedom interpolate between these regimes, provides strong motivation to study its properties as a function of length scale [1].

A tried-and-true method for studying the degrees of freedom of a material as a function of length (energy) scale is to study the attenuation or deflection of an external probe, for example an electron beam, in the material as a function of its energy. The extremely short lifetime of the quark-gluon plasma makes it impossible to study it with external probes, but high-energy processes produced in the collision can be used as probes. Systematic differences in the yield and properties of high-energy processes in heavy-ion collisions compared to a baseline expectation from protonproton collisions has become a critical avenue for studying the quark-gluon plasma produced in heavy-ion collisions (see [2] for a review of relevant measurements).

In this mini-review, I will give a very personal and incomplete view on the status of using jets to understand the structure of the quark-gluon plasma. I will first mention briefly some broad theoretical frameworks which are employed to study jet modification. I will then discuss challenges in the direct interpretation of jet modification measurements, focusing mostly on biases due to jet selection. Based on the difficulty of both modelling and the direct interpretation of data, it is highly desirable to constrain the properties of the quark-gluon plasma as much as possible independently of the features of specific models. I will advocate a more agnostic use of models to diagnose the physics effects at play in particular observables, understand their qualitative impact on measurements, and generate new ideas about how to disentangle multiple effects. I will briefly mention a few examples I find interesting of using models to understand the origin of non-intuitive features in measurements, and finally discuss some recent effort on data-driven approaches to studying the quark-gluon plasma.

\section{Jets in heavy-ion collisions}

In high-energy collisions between protons, there will occasionally be a particularly high momentum-transfer scattering between partons (quarks or gluons) in the incoming protons. Highlyvirtual partons in the final state of this scattering succesively fragment to produce collimated sprays of hadrons at the detector, called jets. By restricting to those events with jets of high transverse momentum $\left(p_{T}\right)$, it is possible to select high momentum-transfer processes for which the QCD coupling is small. Jet production in proton-proton collisions has been studied extensively and in this regime can be calculated in a controlled way using perturbative QCD.

Qualitatively, the goal of a jet is to collect all hadrons that originate from radiation off of the same initiating high-energy ("hard") parton. In this ideal case, summing for example the $p_{T}$ or invariant mass of all hadrons in the jet gives access to the $p_{T}$ or invariant mass of the initiating parton. In practice, asking which hadrons are associated with the hard scattering is not well-defined. At the hadron level a jet must be defined through a "jet algorithm" that identifies clusters of highmomentum hadrons in the detector, for example [3]. Depending on the cone size of jets in this 
algorithm, radiation not from the initiating parton that ends up inside the jet cone is counted as part of the jet, and radiation from the initiating parton that is at large angles relative to the jet axis may not be included in the jet.

In heavy-ion collisions, similar high-energy processes can occur between protons in the incoming nuclei. It should be kept in mind that parton distribution functions for nuclei are different than those for protons, but at high energies it is thought that these effects are small [4]. To the extent that this effect can be neglected, one can think of jets in heavy-ion collisions as being produced as in proton-proton collisions and subsequently modified by the quark-gluon plasma. In this picture, differences in the properties of jets in proton-proton and heavy-ion collisions is a probe of the quark-gluon plasma jets in heavy-ion collisions must pass through.

The theoretical description and modelling of jets in heavy-ion collisions is made difficult by the fact that many scales of momentum transfer may be relevant for describing energy loss of jets in the quark-gluon plasma, including in regimes where the QCD coupling is large and perturbative techniques are not fully controlled. An energetic parton in a finite-temperature medium will experience radiation induced by the medium in addition to radiation it would have experienced in vacuum. The features of this radiation have been studied extensively (see [5] for a review) and provide many critical insights on the features of parton interactions with the plasma. To make contact with phenomenology it is however often necessary to treat a jet not as a parton but as a complicated multi-scale object. In this direction there has been substantial effort to formulate Soft Collinear Effective Theory, which has proven to be a powerful tool to study jets in proton-proton collisions, to account for interactions of jets with the medium [6-9].

A large number of other phenomenological models of jets in heavy-ion collisions are based on Monte Carlo generators for proton-proton collisions (commonly, РутніA) modified to describe interactions with the plasma (for example, [10-14]). These descriptions are convenient because they can efficiently be used to analyze any observable, include a phenomenological model of hadronization, define a jet through a jet algorithm, and can easily incorporate nuclear parton distribution functions. Though strictly the parton shower is only defined in momentum space, in these models the parton shower is typically assigned a (somewhat ad-hoc) spacetime picture to allow it to be interfaced with the spacetime evolution of the quark-gluon plasma, so that energy lost by partons in the shower can depend on local properties of the medium, for example temperature or density. In some variants, energy lost by partons from the shower is redistributed in the medium. Separating hadrons from the medium and from the shower is not well-defined; experimentally it is necessary to have a procedure for subtracting "background" that is not correlated with the jet. Especially jet observables that are sensitive to low-energy radiation and radiation further from the jet axis (for example, the jet mass) can be very sensitive to medium response and the treatment of background subtraction [15] where many models lose control. Recent measurements of large-radius jets $[16,17]$ may provide an exciting opportunity to learn about this difficult regime from data.

\section{Jet selection and the interpretation of modification observables}

Unfortunately, it is not possible to measure jet-by-jet modification directly, since the properties of a jet when it was produced generally cannot be measured. The standard procedure is to compare the statistical properties of jets in heavy-ion collisions to a baseline of jets in proton-proton collisions 
to infer the modification. This gives rise to several non-trivial interpretation issues which will be discussed below (see also [1]).

First, the sample of jets that is probed by proton-proton and heavy-ion measurements are different. Since the properties of jets are modified by the quark-gluon plasma, if the properties before modification are not accessible then any method to select jets will change the sample. Maybe the most important example of this is the selection of jets by their $p_{T}$ : since jets lose energy in the quark-gluon plasma, samples of proton-proton and heavy-ion jets with the same $p_{T}$ had systematically different $p_{T}$ when they were produced. Each heavy-ion jet was produced with a $p_{T}$ that was higher by the amount of its energy loss (which itself may depend on other aspects of its production). Since almost all properties of jets depend strongly on their $p_{T}$, the statistical properties of these samples of jets would look different even in the absence of modification by the plasma. If there is a difference in the distribution of a jet observable between proton-proton and heavy-ion collisions, it is difficult to disentangle whether this arises because the distribution of that observable was already different when the jets were produced, or due in part to modification by the plasma.

In addition, the sample of heavy-ion jets is substantially biased towards those jets that lost as little energy as possible in the quark-gluon plasma. The reason for this is that the probability for producing a jet falls very steeply with $p_{T}$. For a sample of heavy-ion jets with fixed $p_{T}$, this means that there are many more that were initiated by a hard process that was only slightly higher $p_{T}$ and lost a small amount of energy, than that were produced with much higher $p_{T}$ and lost a lot of energy, just because the former were produced in dramatically larger numbers because they are lower $p_{T}$. This bias can have a particularly large (and confusing) impact on jet modification observables, which will be discussed in more detail in the next Section.

These issues can be reduced in a class of processes where a jet is produced with a high-energy electroweak boson like a photon or $Z$-boson. Since the (uncolored) electroweak boson is unmodified by the quark-gluon plasma, its measured $p_{T}$ is a reasonable proxy for the $p_{T}$ of the parton that initiated the recoiling jet. These processes are much more rare than those producing inclusive jets, but have been accessed experimentally (for example, photon-jet [18, 19] and $Z$-jet [20, 21] $p_{T}$ correlations). Both for the higher statistics (and corresponding possibility of more differential measurements) and the increased sensitivity to gluon-initiated jets, however, it remains critical to use inclusive jet events effectively as a probe of the quark-gluon plasma produced in heavy-ion collisions. To do this requires thinking carefully about how to disentangle physical effects from selection biases.

\section{Disentangling competing effects in models}

Especially with the important role of selection biases and other effects which may convolute the interpretation of jet modification measurements, an important role of models can be to use model information to diagnose the physics that a measurement is sensitive to.

One area in which selection biases have entered the qualitative discussion about the interaction of jets with the quark-gluon plasma is in understanding whether jets get narrower or wider due to modification by the plasma. The measured jet shape in heavy-ion collisions [22, 23] is narrower at intermediate radii than for jets in proton-proton collisions in the same $p_{T}$ range. This narrowing can be understood as an increase in the fraction of (typically narrower) quark-initiated jets in heavy-ion 
collisions due to the larger energy loss of gluon-initiated jets [24]. It was shown in [25] and explored in more detail in [26] that this apparent narrowing can occur even if every individual jet widens due to its interaction with the plasma, because of the selection bias. It is also interesting in comparison to the measurement of photon-tagged jet shapes [27], where the selection bias in $p_{T}$ is removed using the photon tag and the narrowing is not observed (though photon+jet sample is also more dominantly quark jets). It has also been argued that a similar effect may impact fragmentation functions [28] and that selection biases may also substantially decrease the sensitivity of jet observables to medium response [29].

It also appears that the energy asymmetry of back-to-back jets, which was originally thought to be due to different path lengths of each jet in the quark-gluon plasma, may require a more delicate interpretation. It was shown in [30] that the generation of this asymmetry need not necessarily be due to path length differences between the back-to-back jets in the medium. Non-intuitively, samples of jets in this model with the same path length have similar asymmetry to those with varying path lengths, suggesting that fluctuations in the energy loss of jets with the same path length could be crucial to the asymmetry. The fact that energy loss fluctuations can be comparably important to path-length differences has also been observed in [31], and a delicate interplay between the path-length dependence and role of fluctuations has also been observed in [32]. The detailed origin of this effect, namely whether the apparent lack of dependence of the asymmetry on path-length is due to it being a much smaller effect jet-by-jet than the effect of energy loss fluctuations, or due to selection biases, remains to be understood.

It has also been pointed out in [33] that the apparent lack of dependence of the jet $R_{A A}$ on the collision energy, despite the larger energy loss anticipated in the hotter plasma produced at higher energies, could be due to simultaneous effects of different spectra and different energy loss at the two energies. This highlights the importance of measuring energy loss in a way that is less sensitive to the vacuum spectra than the $R_{A A}$, for example [34].

\section{Toward interpreting data without models}

Due to the challenges posed by selection biases and the lack of first-principles modelling, there has been substantial recent work towards increasingly model-agnostic and data-driven approaches to learning about the quark-gluon plasma.

The average energy loss of jets as a function of their $p_{T}$ can be measured from the $\left(p_{T^{-}}\right.$ dependent) shift of the jet-production spectrum to lower $p_{T}$ in heavy-ion collisions compared to proton-proton collisions [34]. It was proposed in [34] that this can be used to mitigate the effects of selection biases in jet modification observables by adjusting the $p_{T}$ range of a heavy-ion jet measurement relative to the $p_{T}$ range of the proton-proton measurement to compensate for the average energy loss of jets at that $p_{T}$. Since this procedure only corrects for the average energy loss, the samples of heavy-ion and proton-proton jets that are compared will still not be identical. Regardless, the selection bias due to energy loss is decreased, and differences between comparing proton-proton and heavy-ion jets either with the same $p_{T}$ or with a $p_{T}$ adjusted to account for energy loss can give a benchmark for the magnitude of selection bias effects. It would additionally be interesting to compare this to using Bayesian analysis to extract the average energy loss from fits to $R_{A A}$ for inclusive and photon-tagged jet measurements as in [35]. A potentially exciting area 
for future exploration is understanding how boson+jet and hadron+jet measurements can be used in tandem with inclusive jet measurements to clarify their interpretation [36].

A theoretically-clear probe of the interactions of the quark-gluon plasma with jets is the difference in energy loss between jets initiated by quarks or gluons. To the extent that the quarkgluon plasma sees a jet as an extended object with the color of the initiating parton, gluon jets will lose more energy by a factor of their larger color charge. Deviations from this scaling would suggest that the quark-gluon plasma resolves finer scales within the jet [37]. Unfortunately, jet measurements are generally a mixture of both quark- and gluon-initiated jets, which makes constraining their separate energy loss difficult. The quenching of jets initiated by a b-quark has been measured $[38,39]$ and shows no significant difference compared to the quenching of inclusive jets, which also contain gluon-initiated jets. Particularly at high $p_{T}$ in heavy-ion collisions however, inclusive jets may have quite high quark fraction, which could make this comparison relatively insensitive to gluon energy loss. There has been recent work aiming to use Bayesian analysis to extract $R_{A A}$ separately for quark and gluon jets from experimental data [40] which found large differences in the quenching of quark- and gluon-initiated jets. A recent method for extracting quark and gluon fractions from data using Pyтнia templates, on the hand, has suggested minimal differences between proton-proton and heavy-ion collisions [41]. A method for extracting quark and gluon fractions without using templates has recently been proposed [42] and applied to heavy-ion collisions [43] and may enable future data-driven extraction of quark and gluon jet modification.

Finally, an exciting direction is to seek new observables that probe aspects of the quark-gluon plasma that are not accessible in other measurements or provide new handles on energy loss and modification. For example, it was proposed in [44] that the intermediate $W$ boson in the decay of a top quark makes it possible to change the amount of time a jet interacts with the quark-gluon plasma, which is not possible in other measurements that average over the full time evolution of the quark-gluon plasma.

\section{Concluding remarks and outlook}

Recent years have seen dramatic progress in increasingly differential measurements of a variety of jet observables in heavy-ion collisions. These provide an exciting opportunity to access the short-distance structure of the quark-gluon plasma. In tandem with theoretical developments and improvements in phenomenological modelling, model-agnostic and data-driven approaches provide an important, and complementary, opportunity to cross-check model assumptions and draw modelindependent conclusions where possible.

\section{Acknowledgements}

I would like to thank the Hard Probes 2020 Organizing Committee for the invitation to give an overview of the medium modification of jets in heavy-ion collisions. I gratefully acknowledge valuable discussions with Quinn Brodsky, Yang-Ting Chien, Eliane Epple, Raghav Kunnawalkam Elayavalli, Gian Michele Innocenti, Vit Kucera, Yen-Jie Lee, Aleksas Mazeliauskas, Guilherme Milhano, Lina Necib, Krishna Rajagopal, Jesse Thaler, Urs Wiedemann, Xiaojun Yao, Yi Yin, and Nima Zardoshti. 


\section{References}

[1] W. Busza, K. Rajagopal and W. van der Schee, Heavy Ion Collisions: The Big Picture, and the Big Questions, Ann. Rev. Nucl. Part. Sci. 68 (2018) 339 [1802 . 04801].

[2] M. Connors, C. Nattrass, R. Reed and S. Salur, Jet measurements in heavy ion physics, Rev. Mod. Phys. 90 (2018) 025005 [1705.01974].

[3] M. Cacciari, G.P. Salam and G. Soyez, The anti- $k_{t}$ jet clustering algorithm, JHEP 04 (2008) 063 [0802 . 1189].

[4] ALICE collaboration, Transverse momentum distribution and nuclear modification factor of charged particles in p-Pb collisions at $\sqrt{s_{N N}}=5.02 \mathrm{TeV}$, Phys. Rev. Lett. 110 (2013) 082302 [1210.4520].

[5] Y. Mehtar-Tani, J.G. Milhano and K. Tywoniuk, Jet physics in heavy-ion collisions, Int. J. Mod. Phys. A 28 (2013) 1340013 [1302 .2579].

[6] G. Ovanesyan and I. Vitev, An effective theory for jet propagation in dense QCD matter: jet broadening and medium-induced bremsstrahlung, JHEP 06 (2011) 080 [1103. 1074].

[7] G. Ovanesyan and I. Vitev, Medium-induced parton splitting kernels from Soft Collinear Effective Theory with Glauber gluons, Phys. Lett. B 706 (2012) 371 [1109 . 5619].

[8] V. Vaidya and X. Yao, Transverse momentum broadening of a jet in quark-gluon plasma: an open quantum system EFT, JHEP 10 (2020) 024 [2004 . 11403].

[9] V. Vaidya, Effective Field Theory for jet substructure in heavy ion collisions, 2010.00028.

[10] K. Zapp, G. Ingelman, J. Rathsman, J. Stachel and U.A. Wiedemann, A Monte Carlo Model for 'Jet Quenching', Eur. Phys. J. C 60 (2009) 617 [0804 . 3568].

[11] J. Casalderrey-Solana, D.C. Gulhan, J.G. Milhano, D. Pablos and K. Rajagopal, A Hybrid Strong/Weak Coupling Approach to Jet Quenching, JHEP 10 (2014) 019 [1405 . 3864].

[12] Y. He, T. Luo, X.-N. Wang and Y. Zhu, Linear Boltzmann Transport for Jet Propagation in the Quark-Gluon Plasma: Elastic Processes and Medium Recoil, Phys. Rev. C 91 (2015) 054908 [1503.03313].

[13] Y. Tachibana, N.-B. Chang and G.-Y. Qin, Full jet in quark-gluon plasma with hydrodynamic medium response, Phys. Rev. C 95 (2017) 044909 [1701. 07951].

[14] J. Putschke et al., The JETSCAPE framework, 1903.07706.

[15] R. Kunnawalkam Elayavalli and K.C. Zapp, Medium response in JEWEL and its impact on jet shape observables in heavy ion collisions, JHEP 07 (2017) 141 [1707 . 01539].

[16] CMS collaboration, Measurement of Jet Nuclear Modification Factor in PbPb Collisions at $\sqrt{s_{N N}}=5.02 \mathrm{TeV}$ with CMS, CMS-PAS-HIN-18-014. 
[17] ATLAS collaboration, Measurement of suppression of large-radius jets and its dependence on substructure in $\mathrm{Pb}+\mathrm{Pb}$ at $5.02 \mathrm{TeV}$ by ATLAS detector, ATLAS-CONF-2019-056.

[18] ATLAS collaboration, Measurement of photon-jet transverse momentum correlations in 5.02 $\mathrm{TeV} P b+\mathrm{Pb}$ and pp collisions with ATLAS, Phys. Lett. B 789 (2019) 167 [1809. 07280].

[19] CMS collaboration, Study of jet quenching with isolated-photon+jet correlations in $\mathrm{PbPb}$ and pp collisions at $\sqrt{s_{\mathrm{NN}}}=5.02 \mathrm{TeV}$, Phys. Lett. B 785 (2018) 14 [1711.09738].

[20] CMS collaboration, Study of Jet Quenching with $Z+$ jet Correlations in Pb-Pb and pp Collisions at $\sqrt{s}_{N N}=5.02 \mathrm{TeV}$, Phys. Rev. Lett. 119 (2017) 082301 [1702.01060].

[21] ATLAS collaboration, Medium-induced modification of Z-tagged charged particle yields in $\mathrm{Pb}+\mathrm{Pb}$ collisions at $5.02 \mathrm{TeV}$ with the ATLAS detector, 2008.09811.

[22] CMS collaboration, Modification of Jet Shapes in PbPb Collisions at $\sqrt{s_{N N}}=2.76 \mathrm{TeV}$, Phys. Lett. B 730 (2014) 243 [1310.0878].

[23] CMS collaboration, Jet properties in PbPb and pp collisions at $\sqrt{s_{\mathrm{NN}}}=5.02 \mathrm{TeV}, \mathrm{JHEP} 05$ (2018) 006 [1803.00042].

[24] Y.-T. Chien and I. Vitev, Towards the understanding of jet shapes and cross sections in heavy ion collisions using soft-collinear effective theory, JHEP 05 (2016) 023 [1509.07257].

[25] K. Rajagopal, A.V. Sadofyev and W. van der Schee, Evolution of the jet opening angle distribution in holographic plasma, Phys. Rev. Lett. 116 (2016) 211603 [1602 . 04187].

[26] J. Brewer, K. Rajagopal, A. Sadofyev and W. Van Der Schee, Evolution of the Mean Jet Shape and Dijet Asymmetry Distribution of an Ensemble of Holographic Jets in Strongly Coupled Plasma, JHEP 02 (2018) 015 [1710.03237].

[27] CMS collaboration, Jet Shapes of Isolated Photon-Tagged Jets in Pb-Pb and pp Collisions at $\sqrt{s_{\mathrm{NN}}}=5.02 \mathrm{TeV}$, Phys. Rev. Lett. 122 (2019) 152001 [1809.08602].

[28] P. Caucal, E. Iancu, A. Mueller and G. Soyez, Nuclear modification factors for jet fragmentation, 2005.05852.

[29] J. Brewer, Q. Brodsky and K. Rajagopal, Disentangling Jet Modification, in 10th International Conference on Hard and Electromagnetic Probes of High-Energy Nuclear Collisions: Hard Probes 2020 [2009.03316].

[30] J.G. Milhano and K.C. Zapp, Origins of the di-jet asymmetry in heavy ion collisions, Eur. Phys. J. C 76 (2016) 288 [1512 . 08107].

[31] M.A. Escobedo and E. Iancu, Event-by-event fluctuations in the medium-induced jet evolution, JHEP 05 (2016) 008 [1601.03629].

[32] J. Brewer, A. Sadofyev and W. van der Schee, Jet shape modifications in holographic dijet systems, 1809.10695. 
[33] Y. He, S. Cao, W. Chen, T. Luo, L.-G. Pang and X.-N. Wang, Interplaying mechanisms behind single inclusive jet suppression in heavy-ion collisions, Phys. Rev. C 99 (2019) 054911 [1809.02525].

[34] J. Brewer, J.G. Milhano and J. Thaler, Sorting out quenched jets, Phys. Rev. Lett. 122 (2019) 222301 [1812.05111].

[35] Y. He, L.-G. Pang and X.-N. Wang, Bayesian extraction of jet energy loss distributions in heavy-ion collisions, Phys. Rev. Lett. 122 (2019) 252302 [1808.05310].

[36] J. Casalderrey-Solana, Z. Hulcher, G. Milhano, D. Pablos and K. Rajagopal, Simultaneous description of hadron and jet suppression in heavy-ion collisions, Phys. Rev. C 99 (2019) 051901 [1808.07386].

[37] L. Apolinário, J. Barata and G. Milhano, On the breaking of Casimir scaling in jet quenching, Eur. Phys. J. C 80 (2020) 586 [2003.02893].

[38] CMS collaboration, Evidence of b-Jet Quenching in PbPb Collisions at $\sqrt{s_{N N}}=2.76 \mathrm{TeV}$, Phys. Rev. Lett. 113 (2014) 132301 [1312 .4198].

[39] CMS collaboration, Comparing transverse momentum balance of bet pairs in pp and $\mathrm{PbPb}$ collisions at $\sqrt{s_{\mathrm{NN}}}=5.02 \mathrm{TeV}, \mathrm{JHEP} 03$ (2018) 181 [1802 . 00707].

[40] J.-W. Qiu, F. Ringer, N. Sato and P. Zurita, Factorization of jet cross sections in heavy-ion collisions, Phys. Rev. Lett. 122 (2019) 252301 [1903.01993].

[41] CMS collaboration, Measurement of quark- and gluon-like jet fractions using jet charge in PbPb and pp collisions at $5.02 \mathrm{TeV}$, JHEP 07 (2020) 115 [2004 . 00602].

[42] E.M. Metodiev and J. Thaler, Jet Topics: Disentangling Quarks and Gluons at Colliders, Phys. Rev. Lett. 120 (2018) 241602 [1802 . 00008].

[43] J. Brewer, J. Thaler and A.P. Turner, Data-driven quark and gluon jet modification in heavy-ion collisions, 2008.08596.

[44] L. Apolinário, J.G. Milhano, G.P. Salam and C.A. Salgado, Probing the time structure of the quark-gluon plasma with top quarks, Phys. Rev. Lett. 120 (2018) 232301 [1711.03105]. 